\title{
Open Problems in Quantum Simulation in Ultra-Submicron Devices*
}

\author{
D. K. FERRY ${ }^{\mathrm{a}, \dagger}$ and J. R. BARKER ${ }^{\mathrm{b}}$ \\ ${ }^{a}$ Center for Solid State Electronics Research, Arizona State University, Tempe, AZ 85287-6206; \\ ${ }^{\mathrm{b}}$ Department of Electrical and Electronic Engineering, University of Glasgow, Glasgow G12 8QQ, United Kingdom
}

\begin{abstract}
Quantum transport is becoming more significant as device size shrinks. For example, as device sizes are scaled below $0.1 \mu \mathrm{m}$, the number of impurities becomes quite small, so that they are no longer homogeneously distributed throughout the device volume and the carriers are localized into quantum boxes, in that self-energy corrections produced by locally high carrier densities will lead to quantum dot formation. This leads to the need to discuss transport through an array of such quantum structures. Here, we discuss several issues which must be considered in treating the transport through such devices.
\end{abstract}

Keywords: Quantum transport, device modeling, impurities

\section{INTRODUCTION}

The demand for enhanced performance in VLSI circuits pushes semiconductor devices to miniaturization limits, at which point leading edge devices will employ $0.07 \mu \mathrm{m}$ gate lengths. There is evidence that we can go beyond this size, and many laboratories have produced experimental devices in the $20-30 \mathrm{~nm}$ gate length range. Thus, one can approach sizes that are only $100 \times$ the atomic spacing in the semiconductor. We must treat transport in a quantum mechanical manner, though we would like to extrapolate from present classical approaches, and retain the appealing framework of the latter formalism.
One major problem is caused by the impurity atoms. For these devices, the active region will contain so few dopant atoms that fluctuations in this number will affect device performance. The impurity profiles determine the device configuration, and most assumptions presume that the impurities are randomly dispersed with a uniform doping profile. However, the fluctuation in the number of total particles in the channel for small devices is comparable to the actual number itself. In addition, it can be expected that the devices will exhibit noticeable conductance variations. It is clear that the carrier density in the channel is very inhomogeneous and this will lead to carrier localization in regions approximately $10-20 \mathrm{~nm}$

\footnotetext{
* Work supported in part by ONR and DARPA

${ }^{\dagger}$ Corresponding author.
} 
on a side, because the exchange energy leads to band-gap narrowing in this region. Consequently, these carriers sit in small 3D quantum boxes containing 10-20 electrons per box [1]. Yet most approaches to classical device simulation do not adequately treat this fluctuation in the carrier density and doping, nor indeed the strong Coulomb correlations.

Several issues must be considered in treating the transport through such devices, among which are:

(1) Phase coherence within the array of quantum boxes;

(2) The transition between semi-classical and fully quantum transport, including the ability or inability of the present heuristic methods based on a quantum potential to properly describe charge bunching and tunneling;

(3) The role of the contacts, vis-à-vis the fabricated boundaries, and the actual versus internal boundaries;

(4) Electron correlation due to the small capacitances between such dots, and the possibility of single-electron effects in fluctuations of the overall device;

(5) The possible need to provide decoherence at the source or drain.

Here, we address some of these problems. To begin, we discuss a general quantum formulation, as well as providing an approach that will allow studies of the above questions. We discuss a prototypical quantum dot; and its experimental behavior, before considering the quantum trajectories and possible extension of device descriptions such as the quantum hydrodynamic approach and the quantum potential.

\section{COUPLING THE DEVICE TO THE SYSTEM}

Let us consider an isolated quantum device (e.g., one quantum dot in a real transistor), which is embedded in its environment. The principles are described by the isolated device, its input and output contacts, and the coupling between these parts. The device is described by its Hamiltonian $H_{d}$ and wave function $\Psi_{d}$, while the input and output contacts are described by the Hamiltonians $H_{u}$ and $H_{y}$ and wave functions $\Psi_{u}$ and $\Psi_{y}$, respectively. The Hilbert space for the total wave function is a summation space, rather than a tensor product space which is often used to describe system-environment interactions. That is, there is a complete Hilbert space for each of the wave functions, and the total space is the summation of these three spaces. This choice of space allows us to talk about wave functions localized in the device, or in the contact, or hybridized between them using wave functions isolated in one part of the overall Hilbert space. It is assumed that the Hamiltonian remains separable into parts representing each space plus coupling terms. Hence, $\Psi_{d}, \Psi_{u}$ and $\Psi_{y}$ represent members of the complete Hilbert spaces for those isolated parts of the total system. From some simple manipulations, we can arrive at the transmission matrix from a pure input state to a pure output state

$$
t=\left(H_{d}+A H_{u}^{-1} R\right)^{-1} C H_{u}^{-1} A .
$$

Here, $A, C$ and $R$ are the coupling matrices from the input contact to the device, from the device to the output contact, and from the device back to the input contact, respectively. There are at least three different cases of interest [1].

The case that is most relevant to the present discussion is particular to quantum dots in which the contacts are open quantum point contacts and we cannot ignore $A H_{u}^{-1} R$. The nature of these quantum point contacts is that the modes in the contact region are eigenmodes of the effective waveguide, and this produces significant collimation of the entering particle beam [2]. There is then a significantly different behavior in the dot, and the response is governed by the semiclassical trajectories that are excited in the dot by the waveguide modes. This behavior is clearly seen in both square dots [3] and in stadium (oval) dots that are classically chaotic $[4,5]$, as may be seen in Figure 1. In these dots, in which only a small 


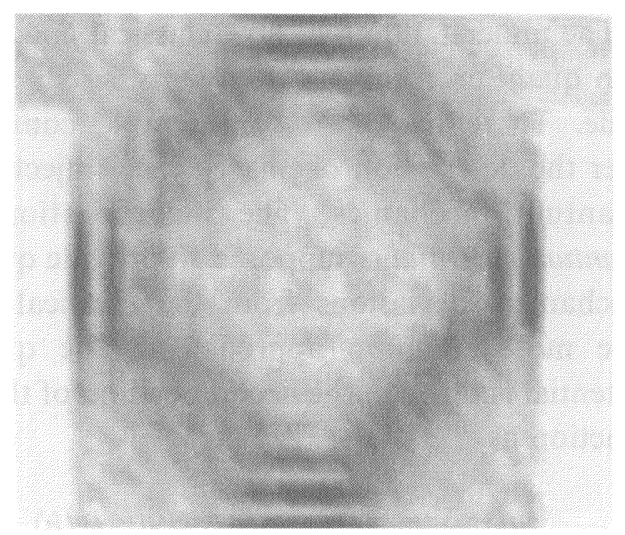

(a)

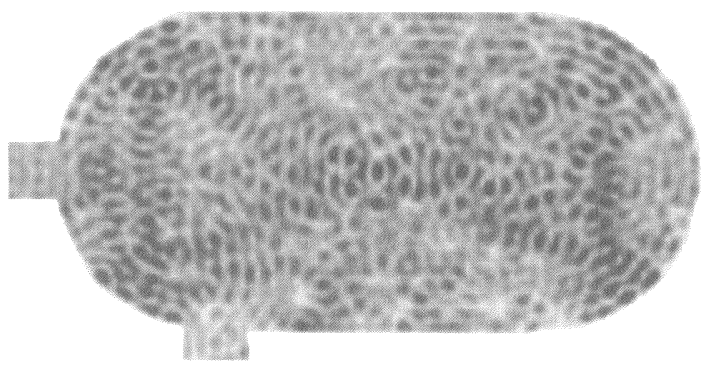

(b)

FIGURE 1 Collimation of the input electrons by the quantum point contact leads to the excitation of heavily scarred wave functions, representative of the regular trajectories in the ballistic quantum dots. Typical scars are shown for a square dot (a) and an oval, or stadium, dot.

family of trajectories is excited by the collimated beam, the spectral density is still evolving from a series of delta functions, and the regular paths give oscillatory contributions to the spectral density. Indeed, measurements of the phase breaking time have clearly shown the $2 \mathrm{D}$ to $0 \mathrm{D}$ transition in the dot as the temperature is lowered (below $130 \mathrm{mK}$ in a 1.0 micron dot) [6]. The periodic orbits combine coherently to produce much stronger oscillations than those of an isolated orbit of a chaotic system. This is important, as the coherence of the phase integrals leads to quite long and periodic orbits. It is the periodic nature of these long orbits, which contributes a large number of terms to the spectral density, corresponding to returns to the input contact after different numbers of traversals. The interference among these waves of successive circuits gives rise to the set of quantized levels that appear in the dot.

The role of the contacts can also be seen in the actual phase-breaking time measured in the dots. In Figure 2, we show measurements on a set of nominally square dots [7]. Here, the phase-breaking time is found to be relatively independent of the dot area, but does depend upon the opening of the QPCs. If the overall resistance is less than 12 $\mathrm{k} \Omega$, the phase-breaking time is about $30 \mathrm{ps}$. On the other hand, if the overall resistance is greater than
$15 \mathrm{k} \Omega$, the phase-breaking time increases by an order of magnitude. We interpret this as an additional phase-breaking process, external to the dot, that is coupled to the dot by a sufficient opening of the QPCs.

What we have found in the above discussion correlates well to what we know from our under-

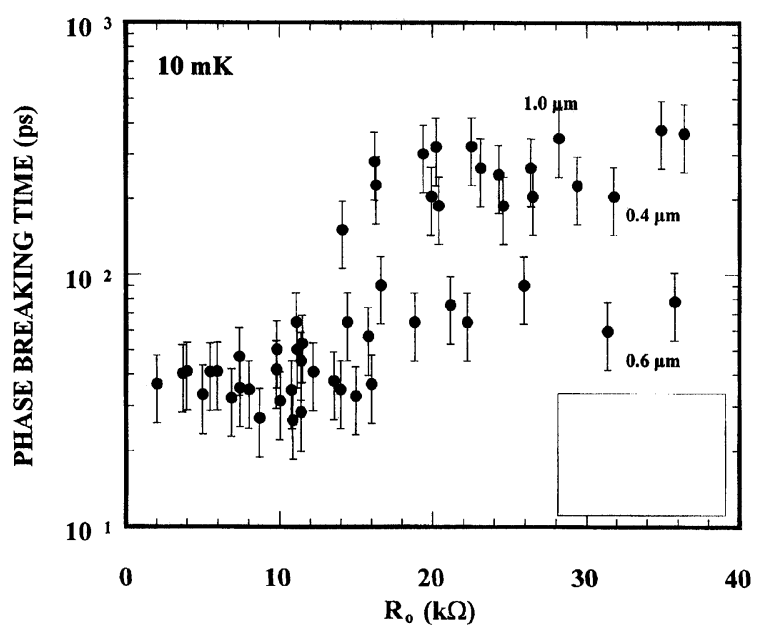

FIGURE 2 The phase breaking time measured experimentally for three different sizes of ballistic quantum dot. The important aspect is that each shows a transition to a much smaller value of the phase breaking time as the quantum point contacts are opened (smaller resistance) so that the dot is more strongly coupled to the environment. 
standing of classical device simulation: The nature of the contacts can dramatically affect, and even dictate, device performance. This point is often missed in discussions of quantum transport which are derived from equilibrium quantum statistical mechanics. The role of the boundaries cannot be overlooked, and usually is the dominant control mechanism in device performance. Without proper treatment of these boundary conditions, no adequate quantum device simulation can be attained.

\section{TRAJECTORIES, PATHS AND POTENTIALS}

Let us now return to the question of the DOS, as measured by the transport in the quantum dot. In square quantum dots, we have found from comparison between theory and experiment that the transport behavior is generally regular. By regular, it is meant that the quasi-ballistic motion scatters elastically off the walls of the dot. In the fully quantum limit of a zero-dimensional dot, the density of states (at $T=0$ ) is simply a sum of delta functions, located at the energy levels of the dot, although the DOS must change to the uniform two-dimensional form as these delta functions are broadened. For a classically chaotic system, it has been thought that opening the quantum billiard (to interactions with the outside world) will introduce chaotic behavior in the level statistics and in the transport. Only recently have the measurements of magnetotransport in fully quantized dots $\left(\Delta \gg k_{B} T\right)$ appeared that show the residual classically regular transport, ruling out the normally accepted chaos theory.

For regular motion, only one family of periods may be observed, but this family will be composed of the fundamental and several harmonics, as seen in the experimental results. We are led to the assertion that the study of transport in "regular" billiards exposes the fundamental quantum structure of the dots themselves, and provides important information as to how the transport will exhibit itself in future electron devices.
The understanding of how classical flows evolve into quantum mechanical currents is still an open issue. There has been considerable controversy over the description of phase space trajectories in quantum mechanics. The incorporation of a quantum potential is supposed to provide quantum mechanical deviations from the classical orbits. The most common approach to the quantum potential is through the decomposition of the wave function as

$$
\Psi(r, t)=\sqrt{\rho(r, t)} \exp (i S(r, t) / \hbar),
$$

where $\rho(r, t)$ is the density ( $\rho$ also will be used as the density matrix later, but this is not its meaning here), $r$ and $t$ are the space-time manifold, and $S(r, t) \hbar$ is the phase associated with the motion.

An unambiguous construction of quantum trajectories, associated with pure states (in both configuration space and phase space) is provided by the Bohm-de Broglie interpretation of quantum mechanics $[8,9]$. Here, the Schrödinger equation for a carrier describes the deterministic, causal evolution of a complex field, but is augmented by the postulate that the carrier may be considered as a classical point particle with position vector $\boldsymbol{x}(t)$ and momentum vector $p(t)$ embedded within the pilot field $\Psi(r, t)$, given by (2). At any time $t_{0}$, the carrier is found with probability density $\rho\left(r, t_{0}\right)$ to have position $\boldsymbol{x}\left(t_{0}\right)=r$ and $\boldsymbol{p}\left(t_{0}\right)=\nabla \boldsymbol{S}\left(r, t_{0}\right)$. Thereafter, the particle trajectory in configuration space and phase space is followed deterministically under the influence of the pilot field via the parameterization

$$
\begin{array}{r}
\boldsymbol{x}(t)=\boldsymbol{x}\left(t_{0}\right)+\int_{t_{0}}^{t} \boldsymbol{p}\left(t^{\prime}\right) d t^{\prime}, \\
\boldsymbol{p}(t)=\nabla S[r(t), t] .
\end{array}
$$

This picture is not a transformation to a particle picture which can replace the quantum description of the system, but instead provides a description in a kinematical sense of the behavior of the system. The evolution of $S$, and hence $p$, is provided by the Schrödinger equation, which is a separate field 
theory. Classically, the action $S$ is determined completely by the underlying particle dynamics in the system, viz. the Hamilton-Jacobi equations. The quantum "action" $S$ is also claimed to be determined by a Hamilton-Jacobi-like equation, which differs from the classical version by coupling to the continuity equation through the quantum potential $V_{Q}$. The latter determines the probability density of locating the particle within quantum theory, as noted previously, and satisfies the continuity equation. However, $S$ is not determined entirely by the local dynamics, but must satisfy a crucial topological condition which follows from its origin as the phase of an independent complex field.

One must be sure to properly incorporate the single-valuedness of the wave function, otherwise the equation of motion for $S$ will not be properly constructed. In particular, we note that $S$ is defined modulo $h$ (Planck's constant). Since, the velocity is normally described from $v=\nabla S / m$, the periodic nature of $S$ leads to the quantization

$$
\oint v \cdot d r=l h / m
$$

where $l$ is an integer. In classical hydrodynamics, the integral on the left is known as the velocity circulation, and is related through Stoke's theorem to the vorticity of the motion. Most approaches to the use of dynamical equations ignore the quantization within the system. The quantization must be put in quite early in the process, as it will not evolve from a classical distribution function.

If we consider any closed contour for which (4) holds, we can write at any point $S^{\prime}=S+N h$. Let us suppose that $N \neq 0$ and that somewhere within the area enclosed by the contour, $S$ goes to zero. This must occur where $\sqrt{\rho}=0$, i.e., at the nodes which are required to preserve the continuity of the wave function itself. At these points, both the real and the imaginary parts of the wave function vanish, and this describes the nodal lines in space. In addition to the quantized orbits (which can be extended to any multiply-connected region), the nodal lines are regions in which the motion is no longer irrotational.
It is important to point out, that closed orbits in a ultrasmall device can be induced not only by reflections between two barriers (the resonant level in the double-barrier tunneling device), but also by multiple scattering from impurities (the source of weak localization and universal conductance fluctuations), or from any combination of these factors. The latter is important for universal conductance fluctuations and, indeed, has been suggested for the observation of weak localization in a single quantum point contact.

One can make a minimalist improvement on the Bohm-deBroglie theory, which is normally described by [10]

$$
\langle\delta(\boldsymbol{x}(t)-r)\rangle=\rho(r, t), \quad \boldsymbol{p}_{0}=\nabla S(r, t) .
$$

We assume that $\boldsymbol{x}(t)$ is a stochastic variable, such that the mean momentum is given by the gradient of the phase as in (5):

$$
\langle\boldsymbol{p}\rangle=\int d^{3} x \rho \nabla S(r, t) .
$$

To account for the stochastic nature, we introduce a momentum fluctuation, which is defined through $\rho \boldsymbol{p}_{1}=\hbar \nabla \rho / 2$. Then, we find a new result for the mean square momentum as

$$
\left\langle p^{2}\right\rangle=\int d^{3} x\left\{\rho\left[p_{0}^{2}+p_{1}^{2}\right]\right\} .
$$

This is the true quantum mechanical expectation value, as compared to the Bohm picture, which omits the term in $p_{1}^{2}$. As noted in [11], this omission leads to a violation of Heisenberg's uncertainty relation. In this extended picture, the equations for continuity and momentum are given by

$$
\begin{gathered}
-\frac{\partial S}{\partial t}=\frac{p_{0}^{2}+p_{1}^{2}}{2 m}+V-\frac{\hbar}{2 m \rho} \nabla \cdot\left(\rho \boldsymbol{p}_{1}\right), \\
\frac{\partial \rho}{\partial t}+\nabla \cdot\left(\frac{\rho \boldsymbol{p}_{0}}{m}\right)=0 .
\end{gathered}
$$

The traditional momentum equation is now found by taking the divergence of (8), but it is clear 
that the quantum potential now splits into two components

$$
V_{Q}=\frac{p_{1}^{2}}{2 m}-\frac{\hbar}{2 m \rho} \nabla \cdot\left(\rho \boldsymbol{p}_{1}\right),
$$

where the leading the term is related to the kinetic energy associated with the quantum fluctuations. The second term in (10) involves the divergence of the fluctuation current and its spatial average over the position probability density vanishes. This is a crucial point, and restores the inherent statistical nature of quantum mechanics to the apparent deterministic form of the Bohm picture. We find that $\boldsymbol{p}_{1}$ satisfies identically a stationary FokkerPlanck equation

$$
\nabla \cdot\left(\frac{\rho p_{1}}{m}\right)=\frac{\hbar}{2 m} \nabla^{2} \rho
$$

reinforcing our view that $\boldsymbol{p}_{1}$ describes the fluctuations. The above equations are an alternative and more acceptable semantic deconstruction [12] of the Schrödinger equation (which must be augmented by the phase boundary condition (4) as before), and points out that there are many different definitions of a "quantum potential".

\section{PROBLEMS OF THE QUANTUM POTENTIAL}

Over and above the above problems of the uniqueness of a quantum potential, the more normally used one has its own problems with any sort of fluctuations. Using the wave function (2) for a pure state, we may easily write two continuity equations for the probability density

$$
\frac{\partial \rho}{\partial t}+\nabla \cdot(\rho v)=0
$$

and for the action

$$
\frac{\partial S}{\partial t}+\frac{(\nabla S)^{2}}{2 m}+V+V_{Q}=0
$$

where

$$
V_{Q}=-\frac{\hbar^{2}}{2 m} \frac{\nabla^{2} \sqrt{\rho}}{\sqrt{\rho}}
$$

is the more usual quantum potential. Taking the gradient of (13) produces an Euler equation which includes the additional force arising from the quantum potential, and this has been the justification for much of the usage of the heuristic quantum hydrodynamic equations for mixed state systems. One must be careful to note the multivalued nature of $S$, quantization of the orbits, and the boundary conditions, scattering, and the continuity of the wave function.

To extend the above approach to a mixed state, one can use the same equations above, but this incorporates errors and approximations. Instead, let us write the density matrix as

$$
\begin{aligned}
\rho\left(r, r^{\prime}, t\right) & =\sum_{n=1}^{N} P_{n}\left|\psi_{n}\right\rangle\left\langle\psi_{n}\right|=\sum_{n=1}^{N} P_{n} \rho_{n}, \\
v_{n} & =\frac{\nabla S_{n}}{m}, \quad \sum_{n=1}^{N} P_{n}=1
\end{aligned}
$$

where, for generality, we do not specify any functional form a priori for the probabilities $P_{n}$ for the states $\psi_{n}$. We may now introduce an average velocity through the prescription

$$
\rho v_{a} \equiv \sum_{n=1}^{N}=P_{n} \rho_{n} v_{n}
$$

for which the continuity Eq. (12) is recovered for the average velocity rather than that of any pure state. We find that a single quantum potential does not immediately appear in the problem, and

$$
\begin{aligned}
m \frac{\partial v_{a}}{\partial t} & +m\left[\sum_{n=1}^{N} P_{n}\left[v_{n} \nabla \cdot\left(\rho_{n} v_{n}\right)+\rho_{n}\left(v_{n} \cdot \nabla\right) v_{n}\right]\right. \\
& \left.-v_{a} \nabla \cdot\left(\rho v_{a}\right)\right]=-\nabla V-\sum_{n=1}^{N} P_{n} \rho_{n} \nabla V_{Q n}
\end{aligned}
$$


Clearly, the "energy" term is quite complex, and not simply related to a single temperature, and even the diagonal terms contain significant fluctuations beyond those related to the velocity fluctuations. The quantum potential is not that of the pure state. Suppose we have a small fluctuation around some steady value, in which we can define the parameters such that

$$
\rho_{n}=\rho+\Delta \rho_{n} \quad\langle\ldots\rangle=\sum_{n=1}^{N} P_{n} \ldots, \quad \rho=\left\langle\rho_{n}\right\rangle .
$$

In this case, the leading correction term is of second order, but we can write the new quantum potential as

$$
\sum_{n=1}^{N} P_{n} \rho_{n} \nabla V_{Q N} \rightarrow \rho \nabla V_{Q}\left(1-\alpha\left\langle\Delta \rho_{n} \Delta \rho_{n}\right\rangle\right)+\ldots
$$

The term in the angular brackets represents a significant contribution to the overall densitydensity correlation function, which is known to produce weak localization and universal conductance fluctuations in mesoscopic devices. Hence, we see that there is a modification of the quantum potential in the mixed state due to these fluctuations, which are readily induced by scattering processes. We can understand the source of the correction term by rewriting (14) as

$$
V_{Q}=-\frac{\hbar^{2}}{2 m} \frac{\nabla^{2} \sqrt{\rho}}{\sqrt{\rho}}=-\frac{\hbar^{2}}{2 m}\left[\frac{\nabla^{2} \rho}{2 \rho}-\left(\frac{\nabla \rho}{2 \rho}\right)^{2}\right]
$$

The second term is directly related to the zeropoint fluctuations, and it is clear that the additional fluctuations introduced by scattering, boundaries, etc., will produce a major contribution to the density-density correlation function through this term. It is the corrections arising from this term that dominantly lead to (19) and, indeed, it is the properties of the second term that are important in introducing the fluctuations of the last section. It is this second term that inspires the introduction of $\boldsymbol{p}_{1}$ in the previous section.

Finally, let us return to the crucial topological condition on the phase of individual quantum states, as expressed through condition (4). This condition achieves enormous complexity for mixed state descriptions, and leads to a condition on the average velocity which takes the form

$$
\Gamma_{a} \oint \boldsymbol{v}_{a} \cdot d \boldsymbol{r}=\oint d \boldsymbol{r} \cdot \sum_{n=1}^{N} P_{n} \frac{\rho_{n}(r) \boldsymbol{v}_{n}(r)}{\rho(r)}
$$

subject to the $N$ conditions

$$
\oint \boldsymbol{v}_{n} \cdot d \boldsymbol{r}=l_{n} h / m
$$

Because the quantum numbers $l_{n}$ are positive or negative integers, there is the possibility that the circulation $\Gamma_{a}$, which is a superposition of terms involving the $l_{n}$, will "phase mix" to zero. If this were exact, the quantum hydrodynamic equations would indeed be simple extensions of classical hydrodynamic theory apart from the quantum potential terms. However, to our knowledge this point has never been explored.

\section{DISCUSSION}

Why has our emphasis been on the concept of defining a quantum kinetic (trajectory based) picture, or even that of a quantum hydrodynamic (streamlines based) picture? As discussed above, experiments are now available on ballistic quantum dots in which one can study both the quantum transport and the classical ballistic transport in a manner that good agreement is achieved between the two and with experiment. Moreover, hydrodynamic approaches have been extensively pursued for "quantum" simulation of small devices. Here, however, we have demonstrated significant limitations and problems in the latter.

Our motivation is to find a true quantum kinetic, or quantum hydrodynamic, picture that 
properly allows for the fluctuations, and recognizes the particular problems which derive from the explicit non-locality of the problem and properly accounts for the topological phase boundary conditions. The success of Monte Carlo suggests that one might analogously find a Monte Carlo, trajectory-based, non-deterministic picture for the Schrödinger equation and/or the densitymatrix Liouville equation.

To this end, we can return to the ballistic quantum dots, as the regular trajectories that exist in these structures provide an ideal testbed for studying the evolution of the classical trajectories into the quantum kinetic picture. Moreover, these structures are explicitly dependent upon the details of the confining boundary potentials and the initial conditions defined by the contacts. They are an ideal structure to study quantum kinetics without the important quantum effects being masked by an overlaying role of inelastic processes, which ultimately destroy much of the quantization.

\section{Acknowledgement}

The authors would like to express their appreciation to Jon Bird for making the data of Figure 3 available prior to publication, and to D. Vasileska, S. M. Goodnick, and A. Asenov for helpful discussions.

\section{References}

[1] Ferry, D. K., Akis, R., Udipi, S., Vasileska, D., Pivin, D. P. Jr., Connolly, K. M., Bird, J. P., Ishibashi, K., Aoyagi, Y., Sugano, T. and Ochiai, Y. (1997). "Carrier Transport in Nanodevices," Jpn. J. Appl. Phys., 36, 1841-5.
[2] Akis, R., Ferry, D. K. and Bird, J. P. (1996). "Magnetotransport fluctuations in regular semiconductor ballistic quantum dots", Phys. Rev. B, 54, 17705-15.

[3] Bird, J. P., Ferry, D. K., Akis, R., Ochiai, Y., Ishibashi, K., Aoyagi, Y. and Sugano, T. (1996). "Periodic conductance fluctuations and stable orbits in mesoscopic semiconductor billiards", Euro-Phys. Lett., 35, 529-34.

[4] Akis, R., Ferry, D. K. and Bird, J. P., "Wave-function scarring effects in stadium-shaped quantum dots", Phys. Rev. Lett..

[5] Okubo, Y., Ochiai, Y., Vasileska, D., Akis, R., Ferry, D. K., Bird, J. P., Ishibashi, K., Aoyagi, Y. and Sugano, T. (1997). "Stability of Regular Orbits in Ballistic Quantum Dots", Phys. Lett. A, in press.

[6] Bird, J. P., Ishibashi, K., Ferry, D. K., Ochiai, Y., Aoyagi, Y. and Sugano, T. (1995). "Phase breaking in ballistic quantum dots: Transition from two- to zero-dimensional behavior", Phys. Rev. B, 51, 18037-40.

[7] Bird, J. P., private communication.

[8] Bohm, D. (1952). "A suggested interpretation of the quantum theory in terms of 'hidden' variables, I and II", Phys. Rev., 85, 166-193.

[9] Holland, P. R. (1993). The Quantum Theory of Motion (Cambridge University Press, Cambridge).

[10] Barker, J. R. (1995). "Trajectories in Quantum Transport", in Quantum Transport in Ultrasmall Devices, Eds. by D. K. Ferry, H. L. Grubin, C. Jacoboni and A.-P. Jauho (Plenum Press, New York), pp. 171-180.

[11] Barker, J. R. (1992). "Fundamental Aspects of Quantum Transport Theory", in Basic Properties of Semiconductors, Vol. 1 of Handbook of Semiconductors, revised, Ed. by P. T. Landsberg (North-Holland, Amsterdam), pp. 1079-1127.

[12] Barker, J. R. (1994). "On the pilot field representation of quantum transport theory", Semicond. Sci. Technol., 9, 911-917.

\section{Authors' Biographies}

David K. Ferry is Regents' Professor of Electrical Engineering at Arizona State University, where he has been since 1983. He is a fellow of the American Physical Society and the Institute of Electrical and Electronics Engineers.

John R. Barker is Professor of Electronics at the University of Glasgow. He is a Fellow of the Royal Society of Edinburgh. 

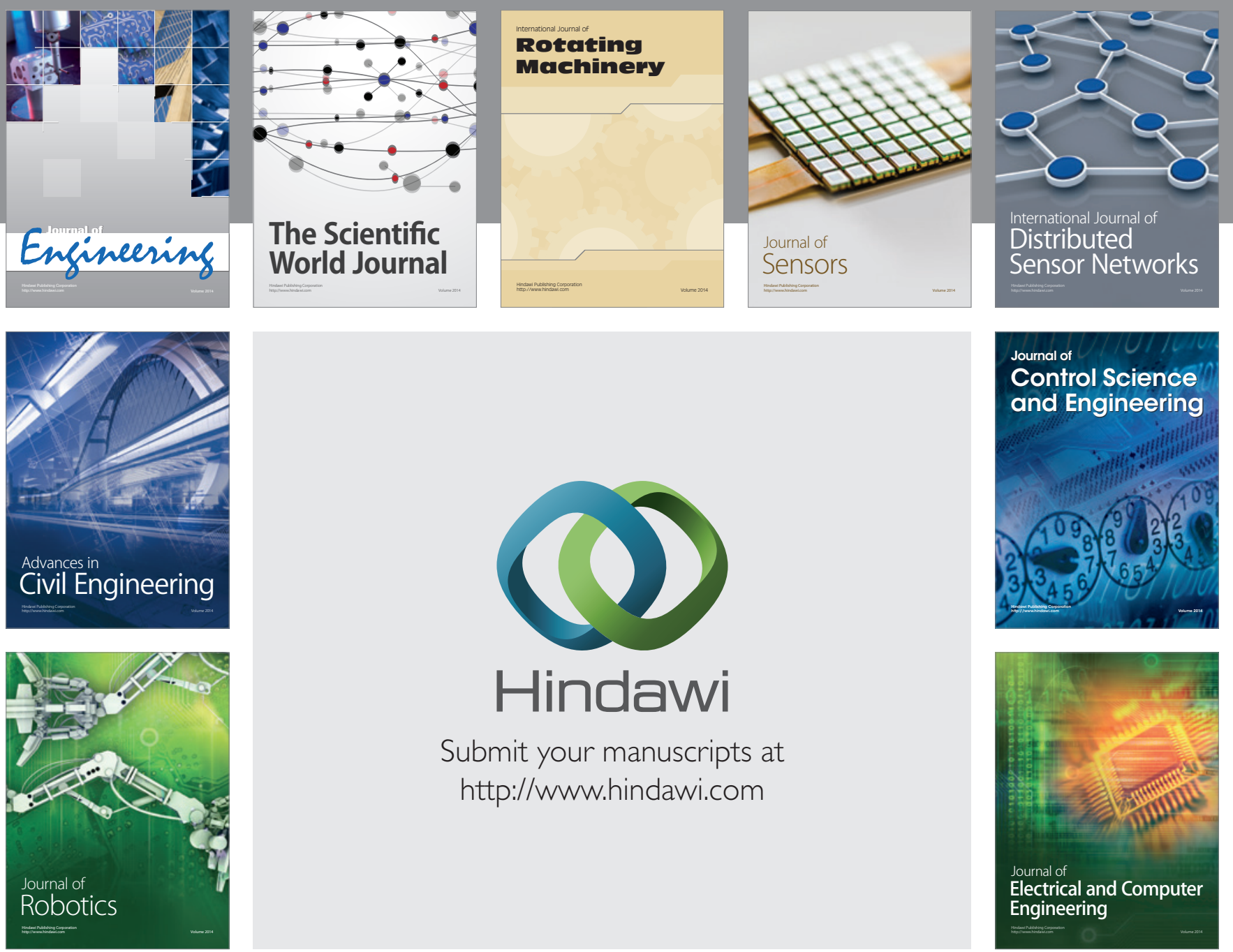

Submit your manuscripts at

http://www.hindawi.com
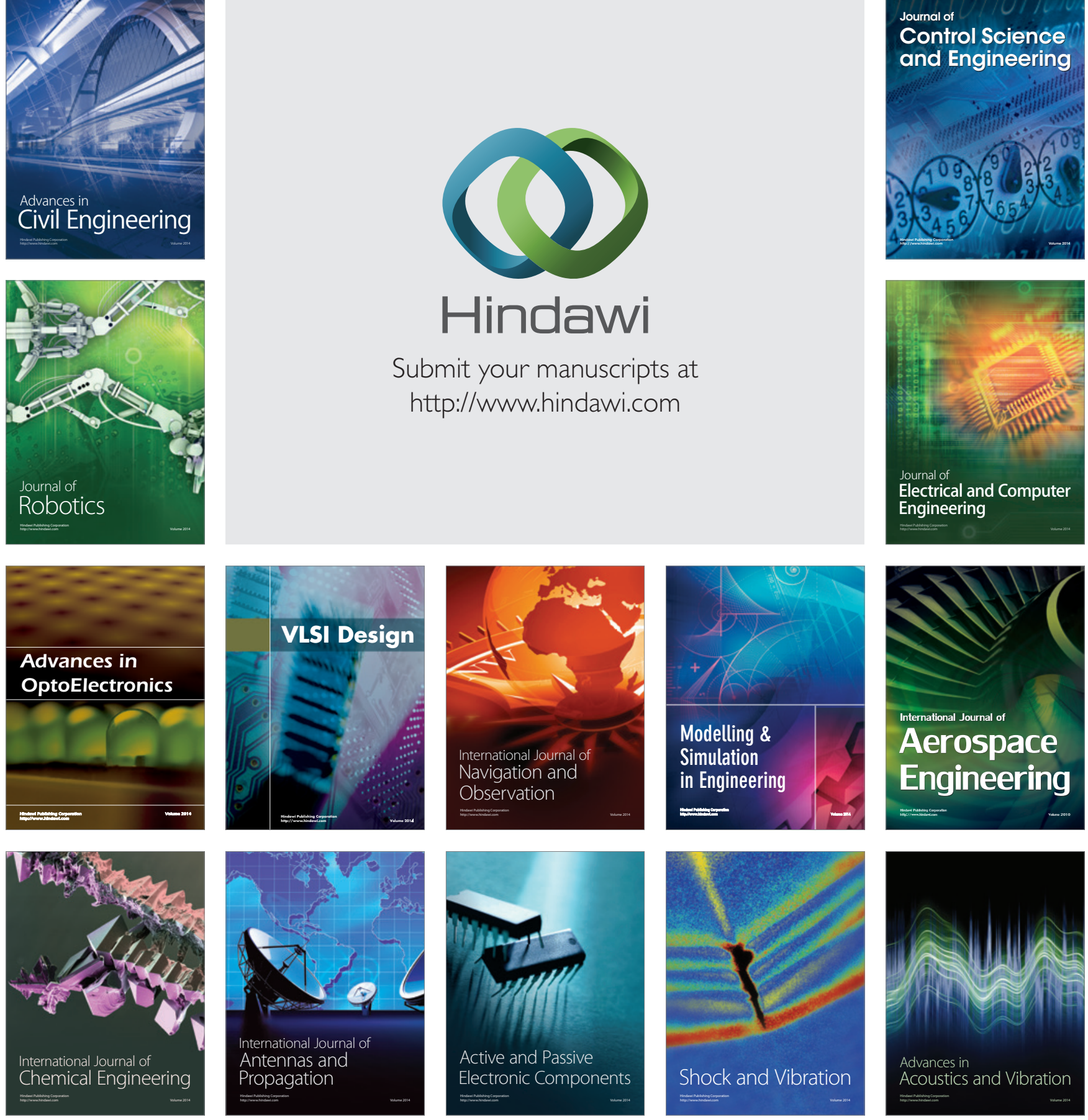